\title{
Research Square \\ Application of Extended Rational Trigonometric Techniques to Investigate Solitary Wave Solutions
}

\author{
Nadia Mahak \\ University of the Punjab \\ ghazala akram ( $\nabla$ toghazala2003@yahoo.com ) \\ University of the Punjab
}

\section{Research Article}

Keywords: Nonlinear partial differential equations, Boiti-Leon-Manna-Pempinelli equation, Exact solutions, Extended rational sine-cosine method, Extended rational sinh-cosh method

Posted Date: June 11th, 2021

DOl: https://doi.org/10.21203/rs.3.rs-595840/v1

License: (1) This work is licensed under a Creative Commons Attribution 4.0 International License. Read Full License

Version of Record: A version of this preprint was published at Optical and Quantum Electronics on July 22nd, 2021. See the published version at https://doi.org/10.1007/s11082-021-03060-1. 


\title{
Application of Extended Rational Trigonometric Techniques to Investigate Solitary Wave Solutions
}

\author{
Nadia Mahak, Ghazala Akram*
}

\begin{abstract}
In this paper, a variety of novel exact traveling wave solutions are constructed for the (2+1)-dimensional Boiti-Leon-Manna-Pempinelli equation via analytical techniques, namely, extended rational sine-cosine method and extended rational sinh-cosh method. The physical meaning of the geometrical structures for some of these solutions is discussed. Obtained solutions are expressed in terms of singular periodic wave, solitary waves, bright solitons, dark solitons, periodic wave and kink wave solutions with specific values of parameters. For the observation of physical activities of the problem, achieved exact solutions are vital. Moreover, to find analytical solutions of the proposed equation many methods have been used but given methodologies are effective, reliable and gave more and novel exact solutions.
\end{abstract}

Keywords: Nonlinear partial differential equations, Boiti-Leon-Manna-Pempinelli equation, Exact solutions, Extended rational sine-cosine method, Extended rational sinh-cosh method.

PACS: 42.65.Wi; 02.30.Jr; 47.35.Fg; 4.20.Jb.

\section{Introduction}

Investigating soliton wave solutions to nonlinear partial differential equations has long been a major impact in the field of mathematical physics. Traveling wave solutions of NPDEs in the type of soliton solutions have essential significance since they create a strong relation between mathematics and physics. Exact traveling wave solutions are considered best to understand the phenomena of natural sciences. A better deal of applications of NPDEs therefore appealed numerous researchers to look for their exact solutions. Many methods have been applied to find exact solutions of NPDEs such as, generalized exponential rational function [1], tanh method [2], the $\exp (-\phi \xi)$-expansion method [3], the extended rational sine-cosine approach and extended rational sinh-cosh approach $[4,5]$, F-expansion method [6], extended Fan sub-equation method [7], the $\left(\frac{G^{\prime}}{G}\right)$-expansion method [8], the first integral method $[9,10]$, the unified method [11], the extended $\left(\frac{G^{\prime}}{G^{2}}\right)$-expansion method [12], the generalised unified method [13], hyperbolic and exponential ansatz methods [14], the Hirota bilinear [15], the ansatz method [16], the modified Kudryashov and new auxiliary equation methods [17], the general bilinear techniques [18], Sine-Gordon expansion method [19], the Hirota method[20], and so on.

${ }^{*}$ Department of Mathematics, University of the Punjab, Lahore 54590, Pakistan. Email: nadiamehboob86@gmail.com and toghazala2003@yahoo.com 
However, the present work focus on the adoption of two novel approaches: the extended rational sinecosine approach and extended rational sinh-cosh approach to seek exact traveling wave solutions of the $(2+1)$-dimensional Boiti-Leon-Manna-Pempinelli equation. The Asymmetric-Nizhnik-NovikovVeselov equation (ANNV) which is actually a two-dimensional KdV equation depicted by the system of equations;

$$
\begin{aligned}
& \phi_{x}-\psi_{y}=0 \\
& \phi_{t}-3(\phi \psi)_{x}+\phi_{x x x}=0
\end{aligned}
$$

and Boiti et al. [21] initially derived the above system that is a model for an incompressible fluid where $\phi$ and $\psi$ are the segments of the dimensionless speed. The system has been generally investigated from different view points, for example, the investigation of its Painlevè property, Lie symmetries, its solutions using arbitrary exponential functions, its the conservation law forms, its exact solution using a separation of variable approach etc. By inserting the transformation: $\psi=\Phi_{x}$ and $\phi=\Phi_{y}$, this system of equations yields

$$
\Phi_{y t}+\Phi_{x x x y}-3 \Phi_{x x} \Phi_{y}-3 \Phi_{x y} \Phi_{x}=0
$$

where $\Phi=\Phi(x, y, t)$ and this equation (1.1) is called as Boiti-Leon-Manna-Pempinelli (BLMP) equation which was derived by Gilson et al. [22] during their researched a $(2+1)$-dimensional generalization of the AKNS shallow-water wave equation using the bilinear method. This equation was utilized to depict the $(2+1)$-dimensional interaction of the Riemann wave propagated along the $y$-axis with a long wave propagated along the $x$-axis. Many researchers are focusing to extract exact solutions of BLMP equation using various different methods such as, based on the binary Bell polynomials [23], Wronskian formalism and the Hirota method [24, 25], the extended homoclinic test approach [26], the rational sine-cosine method [27] and so on.

The strategy of the paper is summarized as follows: Demarcation of extended rational sine-cosine and extended rational sinh-cosh approaches are presented, in Section 2. In Section 3, application of these methods on the BLMP equation is investigated and graphs of some obtained solutions are drawn in Section 4. Conclusion is given in Section 5. Section 6 represents future recommendations.

\section{Algorithms}

Consider the nonlinear partial differential equation (NPDE):

$$
F\left(\Phi, \Phi_{x}, \Phi_{t}, \Phi_{x x}, \Phi_{x t}, \ldots\right)=0
$$

where $\Phi=\Phi(x, t)$ and inserting the following traveling wave transformation

$$
\Phi(x, t)=\Phi(\psi), \quad \psi=x-c t,
$$

where $c$ refers the wave speed, which converts the NPD Eq.(2.2)into an ODE:

$$
G\left(\Phi,-c \Phi^{\prime}, \Phi^{\prime}, c^{2} \Phi^{\prime \prime}, \Phi^{\prime \prime},-c \Phi^{\prime \prime}, \ldots\right)=0
$$

where' denotes the derivative with respect to $\psi$.

\subsection{Extended rational sine-cosine method}

Step 1. To obtain the solutions of Eq.(2.3), extended rational sine-cosine method asserts the general solution in the form

$$
\Phi(\psi)=\frac{\xi_{0} \sin (\mu \psi)}{\xi_{2}+\xi_{1} \cos (\mu \psi)}, \quad \cos (\mu \psi) \neq-\frac{\xi_{2}}{\xi_{1}},
$$


or,

$$
\Phi(\psi)=\frac{\xi_{0} \cos (\mu \psi)}{\xi_{2}+\xi_{1} \sin (\mu \psi)}, \quad \sin (\mu \psi) \neq-\frac{\xi_{2}}{\xi_{1}},
$$

where the unknown parameters $\xi_{0}, \xi_{1}, \xi_{2}$ and $\mu$ is the wave number can be determined later.

Step 2. By substituting Eq.(2.4) or Eq.(2.5) into Eq.(2.3), polynomials in $\cos (\mu \psi)$ or $\sin (\mu \psi)$ are obtained. Then collecting all coefficients with like powers of $\cos (\mu \psi)^{z}$ or $\sin (\mu \psi)^{z}$, (where $z$ is a positive integer) and equating them to zero. A set of algebraic equations can be obtained. The resulting equations are solved with the aid of Maple to get the values of unknown constants $\xi_{0}, \xi_{1}$, $\xi_{2}, c$ and $\mu$.

Step 3. Substituting the obtained unknown values from Step 2 into Eq.(2.4) or Eq.(2.5), the solution of Eq.(2.3) can be found.

\subsection{Extended rational sinh-cosh method}

Step 1. To obtain the solutions of Eq.(2.3), extended rational sinh-cosh method asserts the general solution in the form

$$
\Phi(\psi)=\frac{\xi_{0} \sinh (\mu \psi)}{\xi_{2}+\xi_{1} \cosh (\mu \psi)}, \quad \cosh (\mu \psi) \neq-\frac{\xi_{2}}{\xi_{1}},
$$

or,

$$
\Phi(\psi)=\frac{\xi_{0} \cosh (\mu \psi)}{\xi_{2}+\xi_{1} \sinh (\mu \psi)}, \quad \sinh (\mu \psi) \neq-\frac{\xi_{2}}{\xi_{1}},
$$

where the unknown parameters $\xi_{0}, \xi_{1}, \xi_{2}$ and $\mu$ refers the wave number can be determined later. Step 2. By substituting Eq.(2.6) or Eq.(2.7) into Eq.(2.3), polynomials in $\cosh (\mu \psi) \operatorname{or} \sinh (\mu \psi)$ are obtained. Then collecting all coefficients with like powers of $\cosh (\mu \psi)^{z}$ or $\sinh (\mu \psi)^{z}$, (where $z$ is a positive integer) and equating them to zero. A set of equations can be obtained. The resulting equations are solved with the aid of Maple to get the values of unknown constants $\xi_{0}, \xi_{1}, \xi_{2}, c$ and $\mu$.

Step 3. Substituting the obtained unknown values from Step 2 into Eq.(2.6) or Eq.(2.7), the solution of Eq.(2.3) can be found.

\section{Exact solutions of the Proposed PDE}

The transformation:

$$
\Phi(x, y, t)=U(\psi), \quad \psi=\lambda_{1} x+\lambda_{2} y-c t,
$$

where $\lambda_{1}, \lambda_{2}$ and $c$ are constants, is inserting into Eq.(1.1) and the resulting ODE can be written as

$$
-c \lambda_{2} U^{\prime \prime}+\lambda_{1}^{3} \lambda_{2} U^{\prime \prime \prime \prime}-6 \lambda_{1}^{2} \lambda_{2} U^{\prime} U^{\prime \prime}=0 .
$$

Integrating Eq.(3.9) and setting the constant of integration equals to zero which leads

$$
-c \lambda_{2} U^{\prime}+\lambda_{1}^{3} \lambda_{2} U^{\prime \prime \prime}-3 \lambda_{1}^{2} \lambda_{2}\left(U^{\prime}\right)^{2}=0 .
$$

\subsection{Exact solutions by extended rational sine-cosine method}

Suppose that solution of Eq.(3.10) has the form

$$
U(\psi)=\frac{\xi_{0} \sin (\mu \psi)}{\xi_{2}+\xi_{1} \cos (\mu \psi)} .
$$


Substituting Eq.(3.11) into Eq.(3.10), we get a polynomial in $\cos (\mu \psi)$ and then collecting all coefficient of the like powers of $\cos (\mu \psi)^{z}$ and setting them to zero. The following algebraic equations are obtained:

$$
\begin{aligned}
& \cos (\mu \psi)^{3}: c \lambda_{2} \xi_{2} \xi_{1}{ }^{2}+\lambda_{1}{ }^{3} \mu^{2} \xi_{2} \xi_{1}{ }^{2}=0, \\
& \cos (\mu \psi)^{2}: c \lambda_{2} \xi_{1}{ }^{3}+2 c \lambda_{2} \xi_{2}{ }^{2} \xi_{1}+4 \lambda_{1}{ }^{3} \mu^{2} \xi_{1}{ }^{3}-4 \lambda_{1}{ }^{3} \mu^{2} \xi_{2}{ }^{2} \xi_{1}+3 \lambda_{1}{ }^{2} \xi_{0} \mu a_{2}{ }^{2}=0 \text {, } \\
& \cos (\mu \psi)^{1}: c \lambda_{2} \xi_{2}{ }^{3}+2 c \lambda_{2} \xi_{2} \xi_{1}{ }^{2}-4 \lambda_{1}{ }^{3} \mu^{2} \xi_{2} a_{1}{ }^{2}+\lambda_{1}{ }^{3} \mu^{2} \xi_{2}{ }^{3}+6 \lambda_{1}{ }^{2} \xi_{0} \mu \xi_{2} \xi_{1}=0 \text {, } \\
& \cos (\mu \psi)^{0}: c \lambda_{2} \xi_{2}^{2} \xi_{1}-6 \lambda_{1}^{3} \mu^{2} \xi_{1}^{3}+4 \lambda_{1}^{3} \mu^{2} \xi_{2}{ }^{2} \xi_{1}+3 \lambda_{1}{ }^{2} \xi_{0} \mu \xi_{1}^{2}=0 \text {. }
\end{aligned}
$$

The solutions of above equations are classified as

$$
\text { Case 1. } \mu= \pm \frac{1}{2 \lambda_{1}} \sqrt{-\frac{c \lambda_{2}}{\lambda_{1}}}, \xi_{0}= \pm \sqrt{-\frac{c \lambda_{2}}{\lambda_{1}}} \xi_{1}, \xi_{1}=\xi_{1}, \xi_{2}=0 .
$$

For the case 1 the solutions of Eq.(1.1):

$$
\begin{gathered}
\Phi_{1_{1}}(x, y, t)=\sqrt{-\frac{c \lambda_{2}}{\lambda_{1}}} \tan \left[\frac{1}{2 \lambda_{1}} \sqrt{-\frac{c \lambda_{2}}{\lambda_{1}}}\left(\lambda_{1} x+\lambda_{2} y-c t\right)\right] . \\
\Phi_{1_{2}}(x, y, t)=-\sqrt{-\frac{c \lambda_{2}}{\lambda_{1}}} \tan \left[\frac{1}{2 \lambda_{1}} \sqrt{-\frac{c \lambda_{2}}{\lambda_{1}}}\left(\lambda_{1} x+\lambda_{2} y-c t\right)\right] .
\end{gathered}
$$

Case 2. $\mu= \pm \frac{1}{\lambda_{1}} \sqrt{-\frac{c \lambda_{2}}{\lambda_{1}}}, \xi_{0}= \pm \sqrt{-\frac{c \lambda_{2}}{\lambda_{1}}} \xi_{1}, \xi_{1}= \pm \xi_{2}, \quad \xi_{2}=\xi_{2}$.

For the case 2 the solutions of Eq.(1.1):

$$
\begin{aligned}
& \Phi_{2_{1}}(x, y, t)=\sqrt{-\frac{c \lambda_{2}}{\lambda_{1}}} \frac{\sin \left[\frac{1}{\lambda_{1}} \sqrt{-\frac{c \lambda_{2}}{\lambda_{1}}}\left(\lambda_{1} x+\lambda_{2} y-c t\right)\right]}{1+\cos \left[\frac{1}{\lambda_{1}} \sqrt{-\frac{c \lambda_{2}}{\lambda_{1}}}\left(\lambda_{1} x+\lambda_{2} y-c t\right)\right]} . \\
& \Phi_{2_{2}}(x, y, t)=\sqrt{-\frac{c \lambda_{2}}{\lambda_{1}}} \frac{\sin \left[\frac{1}{\lambda_{1}} \sqrt{-\frac{c \lambda_{2}}{\lambda_{1}}}\left(\lambda_{1} x+\lambda_{2} y-c t\right)\right]}{1-\cos \left[\frac{1}{\lambda_{1}} \sqrt{-\frac{c \lambda_{2}}{\lambda_{1}}}\left(\lambda_{1} x+\lambda_{2} y-c t\right)\right]} . \\
& \Phi_{2_{3}}(x, y, t)=-\sqrt{-\frac{c \lambda_{2}}{\lambda_{1}}} \frac{\sin \left[\frac{1}{\lambda_{1}} \sqrt{-\frac{c \lambda_{2}}{\lambda_{1}}}\left(\lambda_{1} x+\lambda_{2} y-c t\right)\right]}{1+\cos \left[\frac{1}{\lambda_{1}} \sqrt{-\frac{c \lambda_{2}}{\lambda_{1}}}\left(\lambda_{1} x+\lambda_{2} y-c t\right)\right]} . \\
& \Phi_{2_{4}}(x, y, t)=-\sqrt{-\frac{c \lambda_{2}}{\lambda_{1}}} \frac{\sin \left[\frac{1}{\lambda_{1}} \sqrt{-\frac{c \lambda_{2}}{\lambda_{1}}}\left(\lambda_{1} x+\lambda_{2} y-c t\right)\right]}{1-\cos \left[\frac{1}{\lambda_{1}} \sqrt{-\frac{c \lambda_{2}}{\lambda_{1}}}\left(\lambda_{1} x+\lambda_{2} y-c t\right)\right]} .
\end{aligned}
$$

\section{OR}

Suppose that Eq.(3.10) has solution in the form, as

$$
U(\psi)=\frac{\xi_{0} \cos (\mu \psi)}{\xi_{2}+\xi_{1} \sin (\mu \psi)} .
$$


Substituting Eq.(3.13) into Eq.(3.10), we get a polynomial in $\sin (\mu \psi)$ and collecting all coefficients of the like powers of $\sin (\mu \psi)$ and setting them to zero. The following algebraic equations are obtained:

$$
\begin{aligned}
& \sin (\mu \psi)^{3}:-c \lambda_{2} \xi_{1}^{2} \xi_{2}+\lambda_{1}^{3} \mu^{2} \xi_{2} \xi_{1}^{2}=0, \\
& \sin (\mu \psi)^{2}:-2 c \lambda_{2} \xi_{1} \xi_{2}{ }^{2}-c \lambda_{2} \xi_{1}{ }^{3}+4 \lambda_{1}{ }^{3} \mu^{2} \xi_{1}{ }^{3}-4 \lambda_{1}{ }^{3} \mu^{2} \xi_{2}{ }^{2} \xi_{1}+3 \lambda_{1}{ }^{2} \xi_{0} \mu d_{2}{ }^{2}=0, \\
& \sin (\mu \psi)^{1}:-c \lambda_{2} \xi_{2}{ }^{3}-2 c \lambda_{2} \xi_{1}{ }^{2} \xi_{2}+\lambda_{1}{ }^{3} \mu^{2} \xi_{2}{ }^{3}-4 \lambda_{1}{ }^{3} \mu^{2} \xi_{2} \xi_{1}{ }^{2}+6 \lambda_{1}{ }^{2} \xi_{0} \mu \xi_{2} \xi_{1}=0, \\
& \sin (\mu \psi)^{0}:-c \lambda_{2} \xi_{1} \xi_{2}{ }^{2}-6 \lambda_{1}{ }^{3} \mu^{2} \xi_{1}{ }^{3}+4 \lambda_{1}{ }^{3} \mu^{2} \xi_{2}{ }^{2} \xi_{1}+3 \lambda_{1}{ }^{2} \xi_{0} \mu \xi_{1}{ }^{2}=0 .
\end{aligned}
$$

The solutions of above equations are classified as

$$
\text { Case 3. } \quad \mu= \pm \frac{1}{2 \lambda_{1}} \sqrt{\frac{c \lambda_{2}}{\lambda_{1}}}, \quad \xi_{0}=\xi_{0}, \quad \xi_{1}= \pm \sqrt{\frac{\lambda_{1}}{c \lambda_{2}}} \xi_{0}, \quad \xi_{2}=0 .
$$

For the case 3 the solutions of Eq.(3.10):

$$
\begin{gathered}
\Phi_{3_{1}}(x, y, t)=\sqrt{\frac{c \lambda_{2}}{\lambda_{1}}} \cot \left[\frac{1}{2 \lambda_{1}} \sqrt{\frac{c \lambda_{2}}{\lambda_{1}}}\left(\lambda_{1} x+\lambda_{2} y-c t\right)\right] . \\
\Phi_{3_{2}}(x, y, t)=-\sqrt{\frac{c \lambda_{2}}{\lambda_{1}}} \cot \left[\frac{1}{2 \lambda_{1}} \sqrt{\frac{c \lambda_{2}}{\lambda_{1}}}\left(\lambda_{1} x+\lambda_{2} y-c t\right)\right] .
\end{gathered}
$$

Case 4. $\quad \mu= \pm \frac{1}{2 \lambda_{1}} \sqrt{\frac{c \lambda_{2}}{\lambda_{1}}}, \quad \xi_{0}= \pm \sqrt{\frac{c \lambda_{2}}{\lambda_{1}}} \xi_{2}, \quad \xi_{1}= \pm \xi_{2}, \quad \xi_{2}=\xi_{2}$.

For the case 4 the solutions of Eq.(3.10):

$$
\begin{gathered}
\Phi_{4_{1}}(x, y, t)=\sqrt{\frac{c \lambda_{2}}{\lambda_{1}}} \frac{\cos \left[\frac{1}{2 \lambda_{1}} \sqrt{\frac{c \lambda_{2}}{\lambda_{1}}}\left(\lambda_{1} x+\lambda_{2} y-c t\right)\right]}{1+\sin \left[\frac{1}{2 \lambda_{1}} \sqrt{\frac{c \lambda_{2}}{\lambda_{1}}}\left(\lambda_{1} x+\lambda_{2} y-c t\right)\right]} . \\
\Phi_{4_{2}}(x, y, t)=\sqrt{\frac{c \lambda_{2}}{\lambda_{1}}} \frac{\cos \left[\frac{1}{2 \lambda_{1}} \sqrt{\frac{c \lambda_{2}}{\lambda_{1}}}\left(\lambda_{1} x+\lambda_{2} y-c t\right)\right]}{1-\sin \left[\frac{1}{2 \lambda_{1}} \sqrt{\frac{c \lambda_{2}}{\lambda_{1}}}\left(\lambda_{1} x+\lambda_{2} y-c t\right)\right]} . \\
\Phi_{4_{3}}(x, y, t)=-\sqrt{\frac{c \lambda_{2}}{\lambda_{1}}} \frac{\cos \left[\frac{1}{2 \lambda_{1}} \sqrt{\frac{c \lambda_{2}}{\lambda_{1}}}\left(\lambda_{1} x+\lambda_{2} y-c t\right)\right]}{1+\sin \left[\frac{1}{2 \lambda_{1}} \sqrt{\frac{c \lambda_{2}}{\lambda_{1}}}\left(\lambda_{1} x+\lambda_{2} y-c t\right)\right]} . \\
\Phi_{4_{4}}(x, y, t)=-\sqrt{\frac{c \lambda_{2}}{\lambda_{1}}} \frac{\cos \left[\frac{1}{2 \lambda_{1}} \sqrt{\frac{c \lambda_{2}}{\lambda_{1}}}\left(\lambda_{1} x+\lambda_{2} y-c t\right)\right]}{1-\sin \left[\frac{1}{2 \lambda_{1}} \sqrt{\frac{c \lambda_{2}}{\lambda_{1}}}\left(\lambda_{1} x+\lambda_{2} y-c t\right)\right]} .
\end{gathered}
$$

\subsection{Exact solutions by extended rational sinh-cosh method}

Suppose that the traveling wave solution of Eq.(3.10) has the form,

$$
U(\psi)=\frac{\xi_{0} \sinh (\mu \psi)}{\xi_{2}+\xi_{1} \cosh (\mu \psi)}
$$




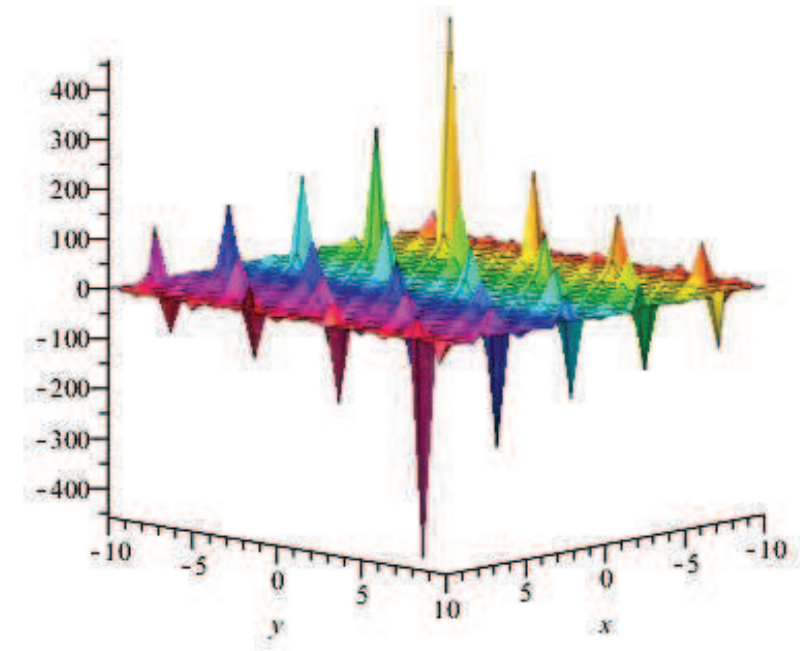

Figure 1: 3D graphics of $\Phi_{1_{1}}$ with $\lambda_{1}=0.09, \lambda_{2}=-1.78$ and $c=0.078$.

Substituting Eq.(3.13) into Eq.(3.10), we get a polynomial in $\cosh (\mu \psi)$ and collecting all terms with the like powers of $\cosh (\mu \psi)^{z}$ and setting them to zero. The following algebraic equations are obtained:

$$
\begin{aligned}
& \cosh (\mu \psi)^{3}: c \lambda_{2} \xi_{2} \xi_{1}{ }^{2}-\lambda_{1}^{3} \mu^{2} \xi_{2} \xi_{1}{ }^{2}=0, \\
& \cosh (\mu \psi)^{2} \quad: \quad 2 c \lambda_{2} \xi_{2}{ }^{2} \xi_{1}+c \lambda_{2} \xi_{1}{ }^{3}-4 \lambda_{1}{ }^{3} \mu^{2} \xi_{1}{ }^{3}+4 \lambda_{1}{ }^{3} \mu^{2} \xi_{2}{ }^{2} \xi_{1}+3 \lambda_{1}{ }^{2} \xi_{0} \mu a_{2}{ }^{2}=0 \\
& \cosh (\mu \psi)^{1}: c \lambda_{2} \xi_{2}{ }^{3}+2 c \lambda_{2} \xi_{2} \xi_{1}{ }^{2}-\lambda_{1}{ }^{3} \mu^{2} \xi_{2}{ }^{3}+4 \lambda_{1}{ }^{3} \mu^{2} \xi_{2} \xi_{1}{ }^{2}+6 \lambda_{1}{ }^{2} \xi_{0} \mu \xi_{2} \xi_{1}=0, \\
& \cosh (\mu \psi)^{0} \quad: \quad c \lambda_{2} \xi_{2}{ }^{2} \xi_{1}-4 \lambda_{1}{ }^{3} \mu^{2} \xi_{2}{ }^{2} \xi_{1}+6 \lambda_{1}{ }^{3} \mu^{2} \xi_{1}{ }^{3}+3 \lambda_{1}{ }^{2} \xi_{0} \mu \xi_{1}{ }^{2}=0 .
\end{aligned}
$$

The solutions of above equations are classified as

$$
\text { Case } 5 . \quad \mu= \pm \frac{1}{2 \lambda_{1}} \sqrt{\frac{c \lambda_{2}}{\lambda_{1}}}, \quad \xi_{0}= \pm \sqrt{\frac{c \lambda_{2}}{\lambda_{1}}} \xi_{1}, \quad \xi_{1}=\xi_{1}, \quad \xi_{2}=0 .
$$

For the case 5 the solutions of Eq.(3.10):

$$
\begin{gathered}
\Phi_{5_{1}}(x, y, t)=\sqrt{\frac{c \lambda_{2}}{\lambda_{1}}} \tanh \left[\frac{1}{2 \lambda_{1}} \sqrt{\frac{c \lambda_{2}}{\lambda_{1}}}\left(\lambda_{1} x+\lambda_{2} y-c t\right)\right] . \\
\Phi_{5_{2}}(x, y, t)=-\sqrt{\frac{c \lambda_{2}}{\lambda_{1}}} \tanh \left[\frac{1}{2 \lambda_{1}} \sqrt{\frac{c \lambda_{2}}{\lambda_{1}}}\left(\lambda_{1} x+\lambda_{2} y-c t\right)\right] .
\end{gathered}
$$

Case 6. $\quad \mu= \pm \frac{1}{\lambda_{1}} \sqrt{\frac{c \lambda_{2}}{\lambda_{1}}}, \quad \xi_{0}= \pm \sqrt{\frac{c \lambda_{2}}{\lambda_{1}}} \xi_{2}, \quad \xi_{1}= \pm \xi_{2}, \quad \xi_{2}=\xi_{2}$. 


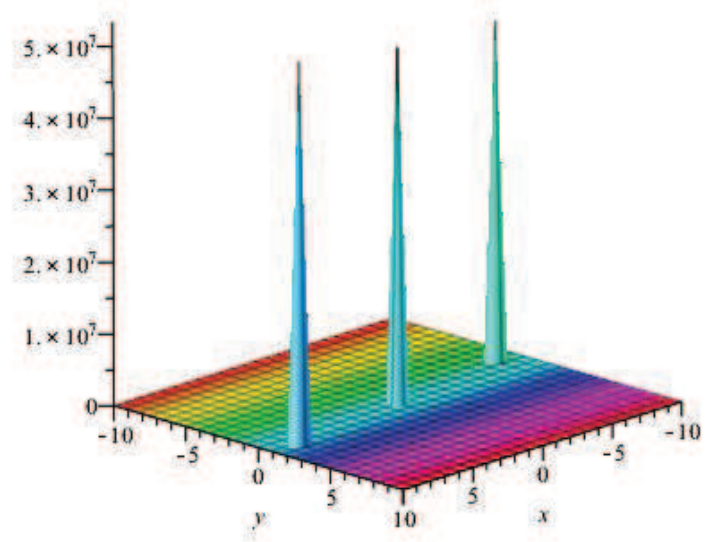

Figure 2: 3D graphics of $\Phi_{2_{4}}$ with $\lambda_{1}=0.09, \lambda_{2}=-0.6$ and $c=0.78$.

For the case 6 the solutions of Eq.(3.10):

$$
\begin{gathered}
\Phi_{6_{1}}(x, y, t)=\sqrt{\frac{c \lambda_{2}}{\lambda_{1}}} \frac{\sinh \left[\frac{1}{\lambda_{1}} \sqrt{\frac{c \lambda_{2}}{\lambda_{1}}}\left(\lambda_{1} x+\lambda_{2} y-c t\right)\right]}{1+\cosh \left[\frac{1}{\lambda_{1}} \sqrt{\frac{c \lambda_{2}}{\lambda_{1}}}\left(\lambda_{1} x+\lambda_{2} y-c t\right)\right]} . \\
\Phi_{6_{2}}(x, y, t)=-\sqrt{\frac{c \lambda_{2}}{\lambda_{1}}} \frac{\sinh \left[\frac{1}{\lambda_{1}} \sqrt{\frac{c \lambda_{2}}{\lambda_{1}}}\left(\lambda_{1} x+\lambda_{2} y-c t\right)\right]}{1+\cosh \left[\frac{1}{\lambda_{1}} \sqrt{\frac{c \lambda_{2}}{\lambda_{1}}}\left(\lambda_{1} x+\lambda_{2} y-c t\right)\right]} . \\
\Phi_{6_{3}}(x, y, t)=\sqrt{\frac{c \lambda_{2}}{\lambda_{1}}} \frac{\sinh \left[\frac{1}{\lambda_{1}} \sqrt{\frac{c \lambda_{2}}{\lambda_{1}}}\left(\lambda_{1} x+\lambda_{2} y-c t\right)\right]}{1-\cosh \left[\frac{1}{\lambda_{1}} \sqrt{\frac{c \lambda_{2}}{\lambda_{1}}}\left(\lambda_{1} x+\lambda_{2} y-c t\right)\right]} . \\
\Phi_{6_{4}}(x, y, t)=-\sqrt{\frac{c \lambda_{2}}{\lambda_{1}}} \frac{\sinh \left[\frac{1}{\lambda_{1}} \sqrt{\frac{c \lambda_{2}}{\lambda_{1}}}\left(\lambda_{1} x+\lambda_{2} y-c t\right)\right]}{1-\cosh \left[\frac{1}{\lambda_{1}} \sqrt{\frac{c \lambda_{2}}{\lambda_{1}}}\left(\lambda_{1} x+\lambda_{2} y-c t\right)\right]} .
\end{gathered}
$$

OR

Suppose that Eq.(3.10) has solution in the form, as

$$
U(\psi)=\frac{\xi_{0} \cosh (\mu \psi)}{\xi_{2}+\xi_{1} \sinh (\mu \psi)} .
$$

Substituting Eq.(3.14) into Eq.(3.10), we get a polynomial in $\sinh (\mu \psi)$ and collecting all terms of the like powers of $\sinh (\mu \psi)^{z}$ and setting them to zero. The following algebraic equations are obtained:

$$
\begin{aligned}
& \sinh (\mu \psi)^{3}: c \lambda_{2} \xi_{1}{ }^{2} \xi_{2}+\lambda_{1}{ }^{3} \mu^{2} \xi_{1}{ }^{2} \xi_{2}=0, \\
& \sinh (\mu \psi)^{2}: 2 c \lambda_{2} \xi_{2}{ }^{2} \xi_{1}-c \lambda_{2} \xi_{1}{ }^{3}-4 \lambda_{1}{ }^{3} \mu^{2} \xi_{1}{ }^{3}-4 \lambda_{1}{ }^{3} \mu^{2} \xi_{2}{ }^{2} \xi_{1}-3 \lambda_{1}{ }^{2} \xi_{0} \mu a_{2}{ }^{2}=0, \\
& \sinh (\mu \psi)^{1}: c \lambda_{2} \xi_{2}{ }^{3}-2 c \lambda_{2} \xi_{1}{ }^{2} \xi_{2}+\lambda_{1}{ }^{3} \mu^{2} \xi_{2}{ }^{3}+4 \lambda_{1}{ }^{3} \mu^{2} \xi_{1}{ }^{2} \xi_{2}+6 \lambda_{1}{ }^{2} \xi_{0} \mu \xi_{2} \xi_{1}=0, \\
& \sinh (\mu \psi)^{0} \quad: \quad-c \lambda_{2} \xi_{2}{ }^{2} \xi_{1}-6 \lambda_{1}{ }^{3} \mu^{2} \xi_{1}{ }^{3}-4 \lambda_{1}{ }^{3} \mu^{2} \xi_{2}{ }^{2} \xi_{1}-3 \lambda_{1}{ }^{2} \xi_{0} \mu \xi_{1}{ }^{2}=0 .
\end{aligned}
$$




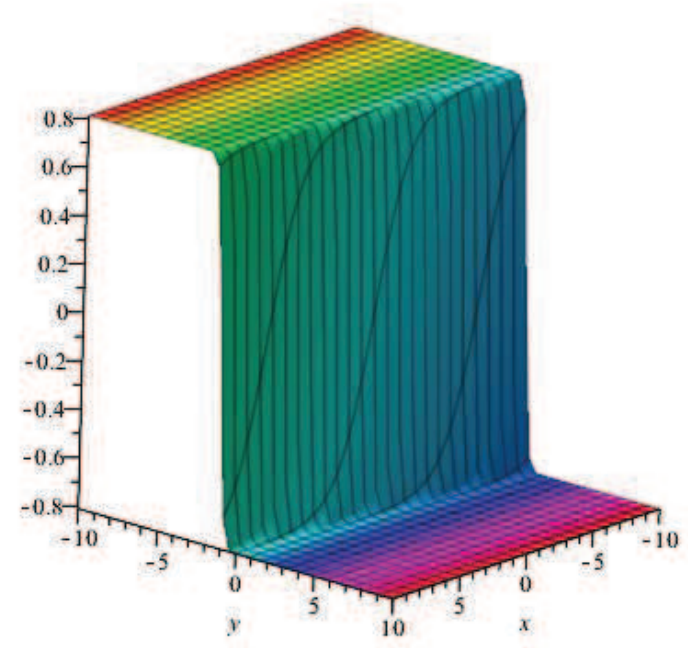

Figure 3: 3D graphics of $\Phi_{5_{2}}$ with $\lambda_{1}=0.09, \lambda_{2}=0.76$ and $c=0.078$.

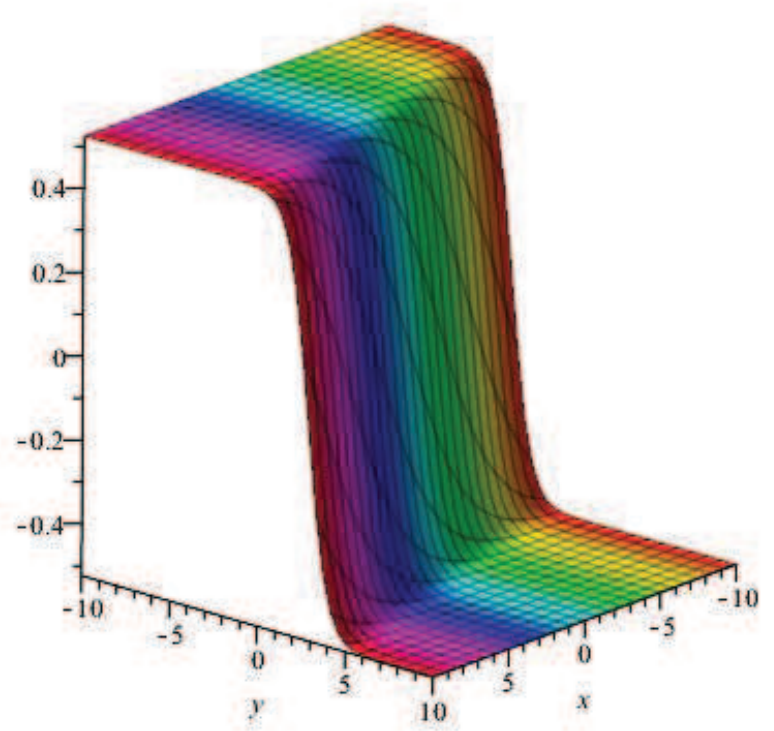

Figure 4: 3D graphics of $\Phi_{6_{1}}$ with $\lambda_{1}=0.5, \lambda_{2}=-1.76$ and $c=0.078$. 
The solutions of above equations are classified as

$$
\text { Case 7. } \mu= \pm \frac{1}{2 \lambda_{1}} \sqrt{-\frac{c \lambda_{2}}{\lambda_{1}}}, \quad \xi_{0}=\xi_{0}, \quad \xi_{1}= \pm \iota \sqrt{\frac{\lambda_{1}}{c \lambda_{2}}} \xi_{0}, \quad \xi_{2}=0 .
$$

For the case 7 the solutions of Eq.(3.10):

$$
\begin{gathered}
\Phi_{7_{1}}(x, y, t)=\sqrt{-\frac{c \lambda_{2}}{\lambda_{1}}} \operatorname{coth}\left[\frac{1}{2 \lambda_{1}} \sqrt{-\frac{c \lambda_{2}}{\lambda_{1}}}\left(\lambda_{1} x+\lambda_{2} y-c t\right)\right] . \\
\Phi_{7_{2}}(x, y, t)=-\sqrt{-\frac{c \lambda_{2}}{\lambda_{1}}} \operatorname{coth}\left[\frac{1}{2 \lambda_{1}} \sqrt{-\frac{c \lambda_{2}}{\lambda_{1}}}\left(\lambda_{1} x+\lambda_{2} y-c t\right)\right] .
\end{gathered}
$$

Case 8. $\quad \mu= \pm \frac{1}{\lambda_{1}} \sqrt{-\frac{c \lambda_{2}}{\lambda_{1}}}, \quad \xi_{0}= \pm \sqrt{\frac{c \lambda_{2}}{\lambda_{1}}} \xi_{2}, \quad \xi_{1}= \pm \iota \xi_{2}, \quad \xi_{2}=\xi_{2}$.

For the case 8 the solutions of Eq.(3.10):

$$
\begin{aligned}
\Phi_{8_{1}}(x, y, t) & =\sqrt{-\frac{c \lambda_{2}}{\lambda_{1}}} \frac{\cosh \left[\frac{1}{\lambda_{1}} \sqrt{-\frac{c \lambda_{2}}{\lambda_{1}}}\left(\lambda_{1} x+\lambda_{2} y-c t\right)\right]}{\sinh \left[\frac{1}{\lambda_{1}} \sqrt{-\frac{c \lambda_{2}}{\lambda_{1}}}\left(\lambda_{1} x+\lambda_{2} y-c t\right)\right]} . \\
\Phi_{8_{2}}(x, y, t) & =-\sqrt{-\frac{c \lambda_{2}}{\lambda_{1}}} \frac{\cosh \left[\frac{1}{\lambda_{1}} \sqrt{-\frac{c \lambda_{2}}{\lambda_{1}}}\left(\lambda_{1} x+\lambda_{2} y-c t\right)\right]}{1+\iota \sinh \left[\frac{1}{\lambda_{1}} \sqrt{-\frac{c \lambda_{2}}{\lambda_{1}}}\left(\lambda_{1} x+\lambda_{2} y-c t\right)\right]} . \\
\Phi_{8_{3}}(x, y, t)= & \sqrt{-\frac{c \lambda_{2}}{\lambda_{1}}} \frac{\cosh \left[\frac{1}{\lambda_{1}} \sqrt{-\frac{c \lambda_{2}}{\lambda_{1}}}\left(\lambda_{1} x+\lambda_{2} y-c t\right)\right]}{1-\iota \sinh \left[\frac{1}{\lambda_{1}} \sqrt{-\frac{c \lambda_{2}}{\lambda_{1}}}\left(\lambda_{1} x+\lambda_{2} y-c t\right)\right]} . \\
\Phi_{8_{4}}(x, y, t)= & -\sqrt{-\frac{c \lambda_{2}}{\lambda_{1}}} \frac{\cosh \left[\frac{1}{\lambda_{1}} \sqrt{-\frac{c \lambda_{2}}{\lambda_{1}}}\left(\lambda_{1} x+\lambda_{2} y-c t\right)\right]}{1-\iota \sinh \left[\frac{1}{\lambda_{1}} \sqrt{-\frac{c \lambda_{2}}{\lambda_{1}}}\left(\lambda_{1} x+\lambda_{2} y-c t\right)\right]} .
\end{aligned}
$$

\subsection{Results and discussion}

Some obtained exact rational trigonometric solutions of the $(2+1)$-dimensional Boiti-Leon-MannaPempinelli equation are shown by graphs along with the physical explanations which are plotted only for $-10 \leq x \leq 10,-10 \leq y \leq 10$ and $t=0$. Eq.(3.12) represents the periodic wave solutions, as tangent is a periodic function with period $2 \pi$. Fig.(1) illustrates the evolution of singular periodic wave solutions for $\Phi_{1_{1}}(x, y, t)$ for $\lambda_{1}=0.09, \lambda_{2}=-1.78$ and $c=0.078$. Taking $\lambda_{1}=0.09, \lambda_{2}=-0.6$ and $c=0.78$, Fig.(2) shows periodic solutions for $\Phi_{2_{4}}(x, y, t)$. Eq.(3.13) shows dark soliton solutions. Further, with $\lambda_{1}=0.09, \lambda_{2}=0.76$ and $c=0.078$, Fig.(3) of $\Phi_{5_{2}}(x, y, t)$ describes kink wave solutions as Kink waves are solitons that rise or descend from one asymptotic state to another. With $\lambda_{1}=0.5$, $\lambda_{2}=-1.76$ and $c=0.078$, Fig.(4) also represents kink type wave solutions for $\Phi_{6_{1}}(x, y, t)$. With the supposition that $\lambda_{1}=-0.5, \lambda_{2}=0.06$ and $c=3$, part $(a)$ of Fig.(5) illustrates the graph of the real part of $\Phi_{8_{1}}(x, y, t)$ which represents bright soliton wave solutions, while part $(b)$ of Fig.(5) shows the graph of the imaginary part of $\Phi_{8_{1}}(x, y, t)$ which describes kink wave solutions. 
(a)
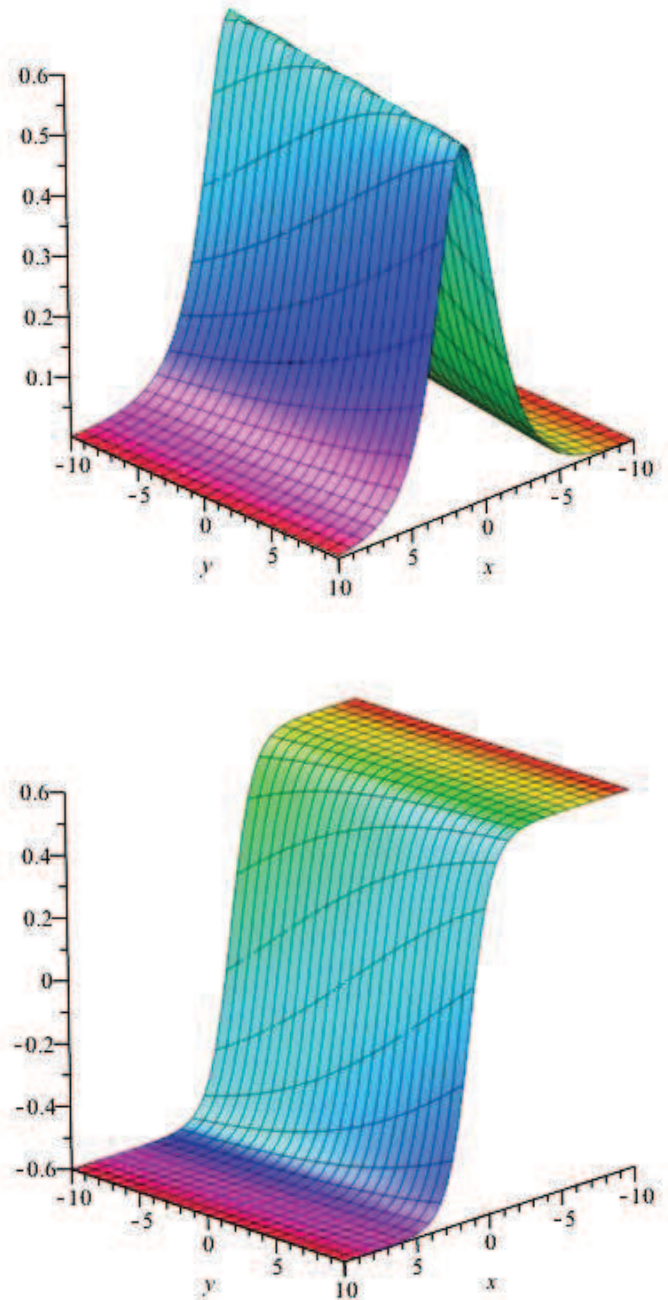

(b)

Figure 5: 3D graphics of real and imaginary parts of $\Phi_{8_{1}}$ with $\lambda_{1}=-0.5, \lambda_{2}=0.06$ and $c=3$. 


\section{Conclusion}

Exact rational trigonometric solutions of the $(2+1)$-dimensional Boiti-Leon-Manna-Pempinelli equation have been retrieved by using extended rational sine-cosine and extended rational sinh-cosh methods. Obtained solitary wave solutions are extremely useful in the study of NPDEs in the context of shallow water waves. Some new graphical representations are obtained with the help of these methods. It is found that some of the obtained exact solutions have likely comparable with [27]. As solutions of Eq.(47) and Eq.(59) in [27] are likely similar with our solutions Eq.(3.13) and Eq.(3.12) respectively. To our knowledge, remaining obtained solutions such as solitary waves, singular periodic wave, bright soltiton, dark soliton, periodic wave and kink wave solutions are new and novel.

\section{Future recommendations}

This paper obtained solitary wave solutions to a nonlinear evolution equation that appears in mathematical physics. These solutions are going to be indeed valuable for conducting future research in this field. One novel future aspect is to consider high dimensional equations for examples with perturbation term(s), fractional temporal evolutions will lead to additional interesting results that will be further closer to realistic situations. Seeking analytical solutions for such equations will be a daunting task. It is believed that the proposed methods are powerful, effective, and may play an important role describing the physical features of various nonlinear complex models.

\section{Declarations}

Funding Not applicable.

Conflicts of interest The Authors have no conflict of interest.

Availability of data and material Not applicable.

Code availability The computations involved in the work are done with the help of Maple and Mathematica.

\section{References}

[1] Osman. M. S, Ghanbari. B. New optical solitary wave solutions of Fokas-Lenells equation in presence of perturbation terms by a novel approach. Optik, 175, 328-333(2018).

[2] Tariq. H, Akram. G. New approach for exact solutions of time fractional Cahn-Allen equation and time fractional Phi-4 equation. Physica. A, 473, 352-362(2017).

[3] Sajid. N, Akram. G. The application of the $\exp (-\phi \xi)$-expansion method for finding the exact solutions of two integrable equations. Math. Probl. Eng, 2018, 1-10(2018).

[4] Mahak. N, Akram. G. Extension of rational sine-cosine and rational sinh-cosh techniques to extract solutions for the perturbed NLSE with Kerr law nonlinearity. Eur. Phys. J. Plus, 134(40), 159-168(2019).

[5] Mahak. N, Akram. G. Exact solitary wave solutions by extended rational sine-cosine and extended rational sinh-cosh techniques. Phys Scripta, 94(11), 115212(2019).

[6] Liu. J, Yang. K. The extended F-expansion method and exact solutions of nonlinear PDEs. Chaos. Solitons. Fract, 22(1), $111-121(2004)$. 
[7] Batool. F, Akram. G. Application of extended Fan sub-equation method to (1+1)-dimensional nonlinear dispersive modified Benjamin-Bona-Mahony equation with fractional evolution. Opt. Quant. Electron, 49(11), 1-9(2017).

[8] Liu, J. G., Osman, M. S., Wazwaz, A. M. A variety of nonautonomous complex wave solutions for the $(2+1)$-dimensional nonlinear Schrödinger equation with variable coefficients in nonlinear optical fibers. Optik, 180, 917-923 (2019).

[9] Akram. G, Mahak. N. Analytical solution of the Korteweg-de Vries equation and microtubule equation using the first integral method. Opt. Quant. Electron 50, 145(2018).

[10] Akram. G, Mahak. N. Application of the first integral method for solving $(1+1)$ dimensional cubic-quintic complex Ginzburg-Landau equation. Optik, 164, 210-217(2018).

[11] Osman, M. S., Rezazadeh, H., Eslami, M., Neirameh, A., Mirzazadeh, M. Analytical study of solitons to benjamin-bona-mahony-peregrine equation with power law nonlinearity by using three methods. University Politehnica of Bucharest Scientific Bulletin-Series A-Applied Mathematics and Physics, 80(4), 267-278 (2018).

[12] Akram. G, Mahak. N. Traveling wave and exact solutions for the perturbed nonlinear Schrödinger equation with Kerr law nonlinearity. Eur. Phys. J. Plus, 133(6), 212-220(2018).

[13] Osman, M. S. Multi-soliton rational solutions for quantum Zakharov-Kuznetsov equation in quantum magnetoplasmas. Wave Random Complex, 26(4), 434-443 (2016).

[14] Ali, K. K., Cattani, C., Gòmez-Aguilar, J. F., Baleanu, D., Osman, M. S. Analytical and numerical study of the DNA dynamics arising in oscillator-chain of Peyrard-Bishop model. Chaos. Solitons. Fract, 139, 110089 (2020).

[15] Liu, J. G., Zhu, W. H., Osman, M. S., Ma, W. X. An explicit plethora of different classes of interactive lump solutions for an extension form of 3D-Jimbo-Miwa model. Eur. Phys. J. Plus, $135(5), 412$ (2020).

[16] Osman, M. S., Liu, J. G., Hosseini, K., Yusuf, A. Different wave structures and stability analysis for the generalized (2+1)-dimension Camassa-Holm-Kadomtsev-Petviashvili equation. Phys Scripta, 95(3), 035229(2020).

[17] Kumar, D., Park, C., Tamanna, N., Paul, G. C., Osman, M. S. Dynamics of two-mode SawadaKotera equation: Mathematical and graphical analysis of its dual-wave solutions. Results Phys, 19, 10358 (2020).

[18] Liu, J. G., Osman, M. S., Zhu, W. H., Zhou, L., Baleanu, D. The general bilinear techniques for studying the propagation of mixed-type periodic and lump-type solutions in a homogenousdispersive medium. AIP Adv, 10(10), 105325 (2020).

[19] Ali, K. K., Osman, M. S., Abdel-Aty, M. New optical solitary wave solutions of Fokas-Lenells equation in optical fiber via Sine-Gordon expansion method. Alexandria Engineering Journal, $59(3), 1191-1196$ (2020).

[20] Ismael, H. F., Bulut, H., Park, C., Osman, M. S. M-lump, N-soliton solutions, and the collision phenomena for the $(2+1)$-dimensional Date-Jimbo-Kashiwara-Miwa equation. Results Phys, 19, 103329 (2020).

[21] Boiti. M, Leon. J, Manna M, Pempinelli F. On the spectral transform of a Korteweg-de Vries equation in two spatial dimension. Inverse. Probl. 2, 271-279(1986).

[22] Gilson. C. R, Nimmo. J. J. C, Willox. R. A (2+1)- dimensional generalization of the AKNS shallow water wave equation. Phys. Lett. A 180, 337-345(1993). 
[23] Luo. L. New exact solutions and Bäcklund transformation for Boiti-Leon-Manna-Pempinelli equation. Phys. Lett. A 375, 1059-1063 (2001).

[24] Delisle. L, Mosaddeghi M. Classical and SUSY solutions of the Boiti-Leon-Manna-Pempinelli equation. J. Phys. A Math. Theor. 46, 115-203 (2013).

[25] Najafi. M, Najafi. M, Arbabi. S. Wronskian determinant solutions of the (2+1)-dimensional Boiti-Leon-Manna- Pempinelli equation. Int. J. Adv. Math. Sci, 1, 8-11 (2013).

[26] Tang. Y, Zai. W. New periodic-wave solutions for $(2+1)$ - and $(3+1)$-dimensional Boiti-LeonManna-Pempinelli equations. Nonlinear. Dynam, 81(1-2), 249-55(2015).

[27] Arbabi, S., Najafi, M. Soliton solutions of nonlinear evolution equations in mathematical physics. Optik, 127(10), 4270-4274(2016). 
Figures

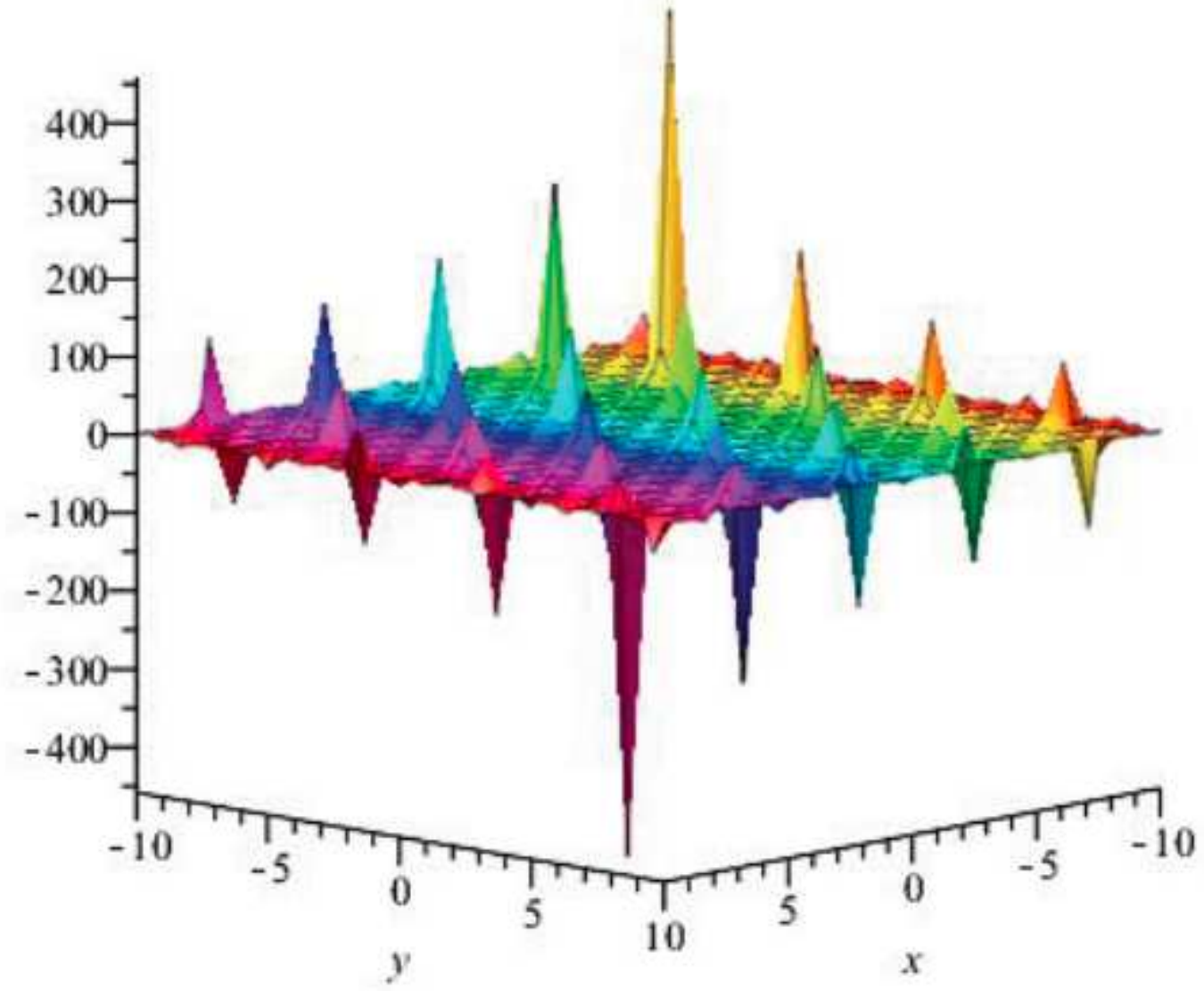

Figure 1

Please see the Manuscript PDF file for the complete figure caption 


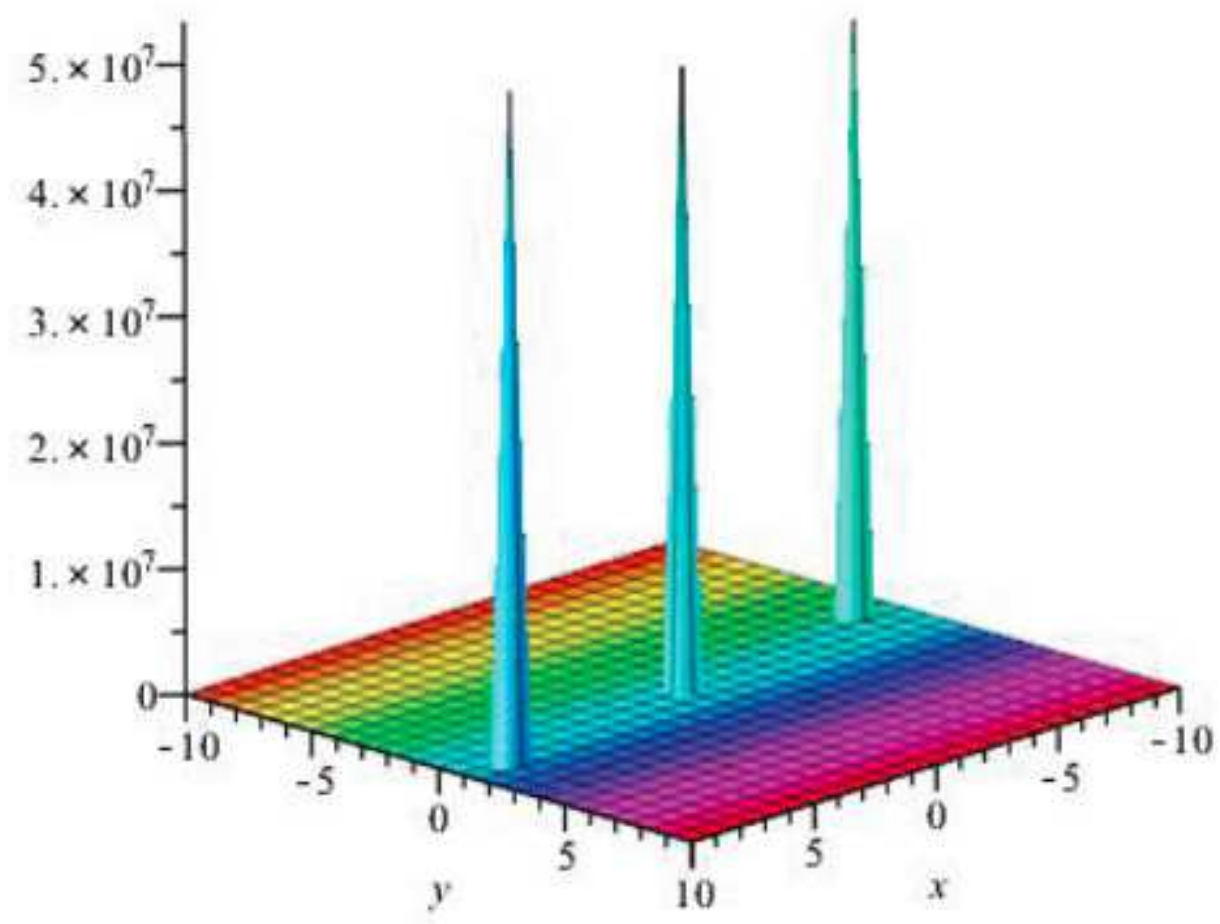

Figure 2

Please see the Manuscript PDF file for the complete figure caption 


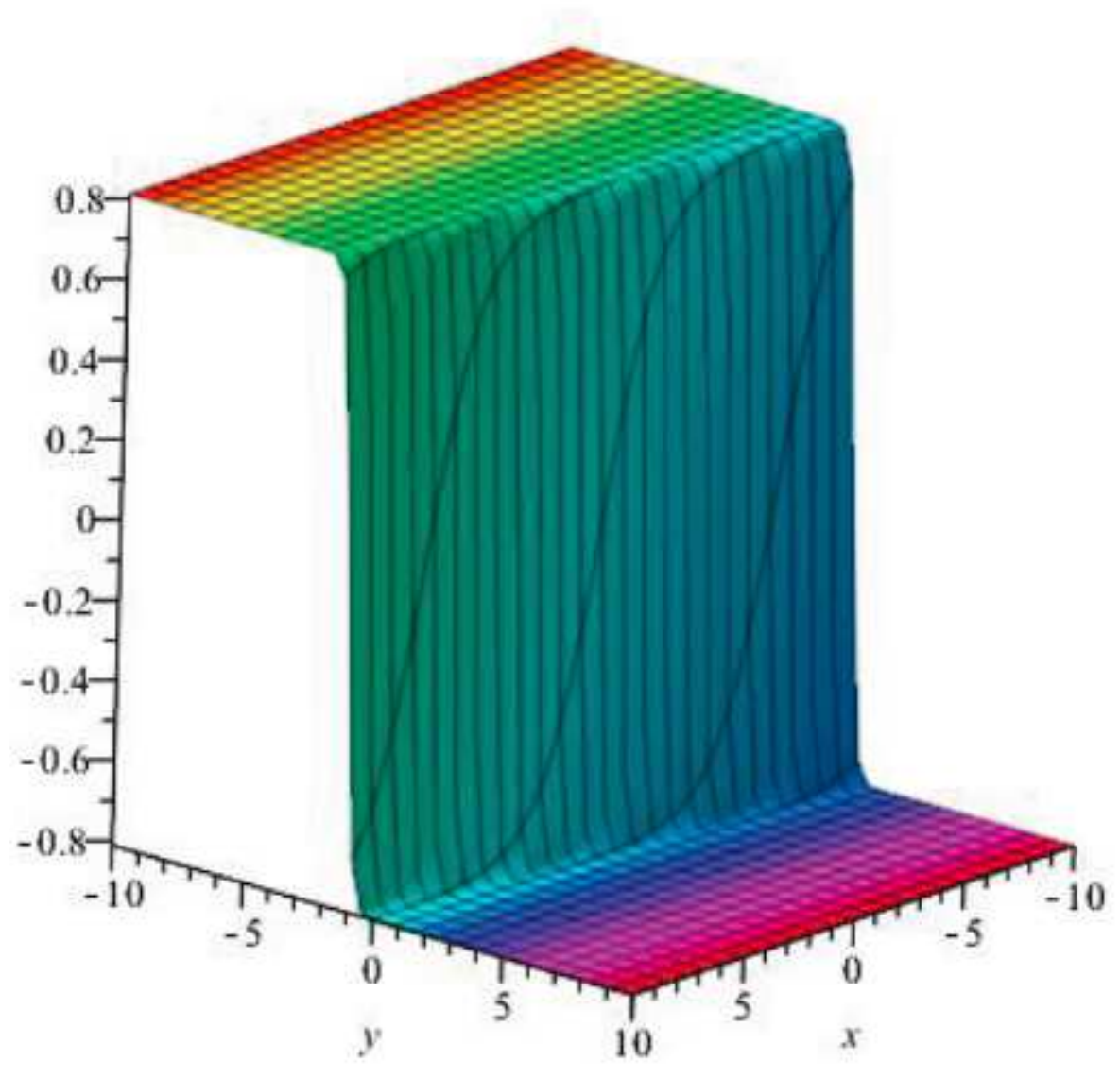

Figure 3

Please see the Manuscript PDF file for the complete figure caption 


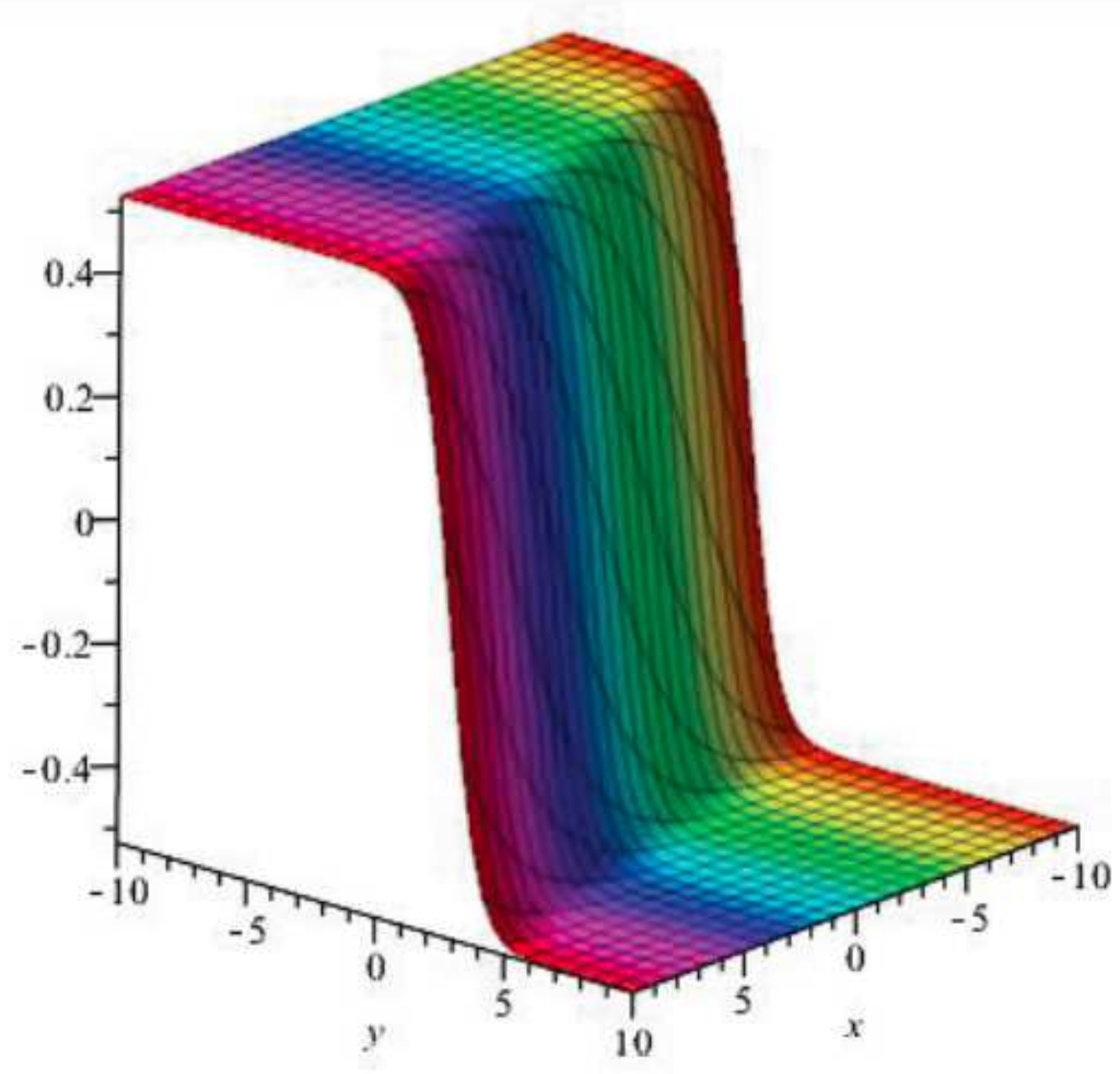

Figure 4

Please see the Manuscript PDF file for the complete figure caption 
(a)
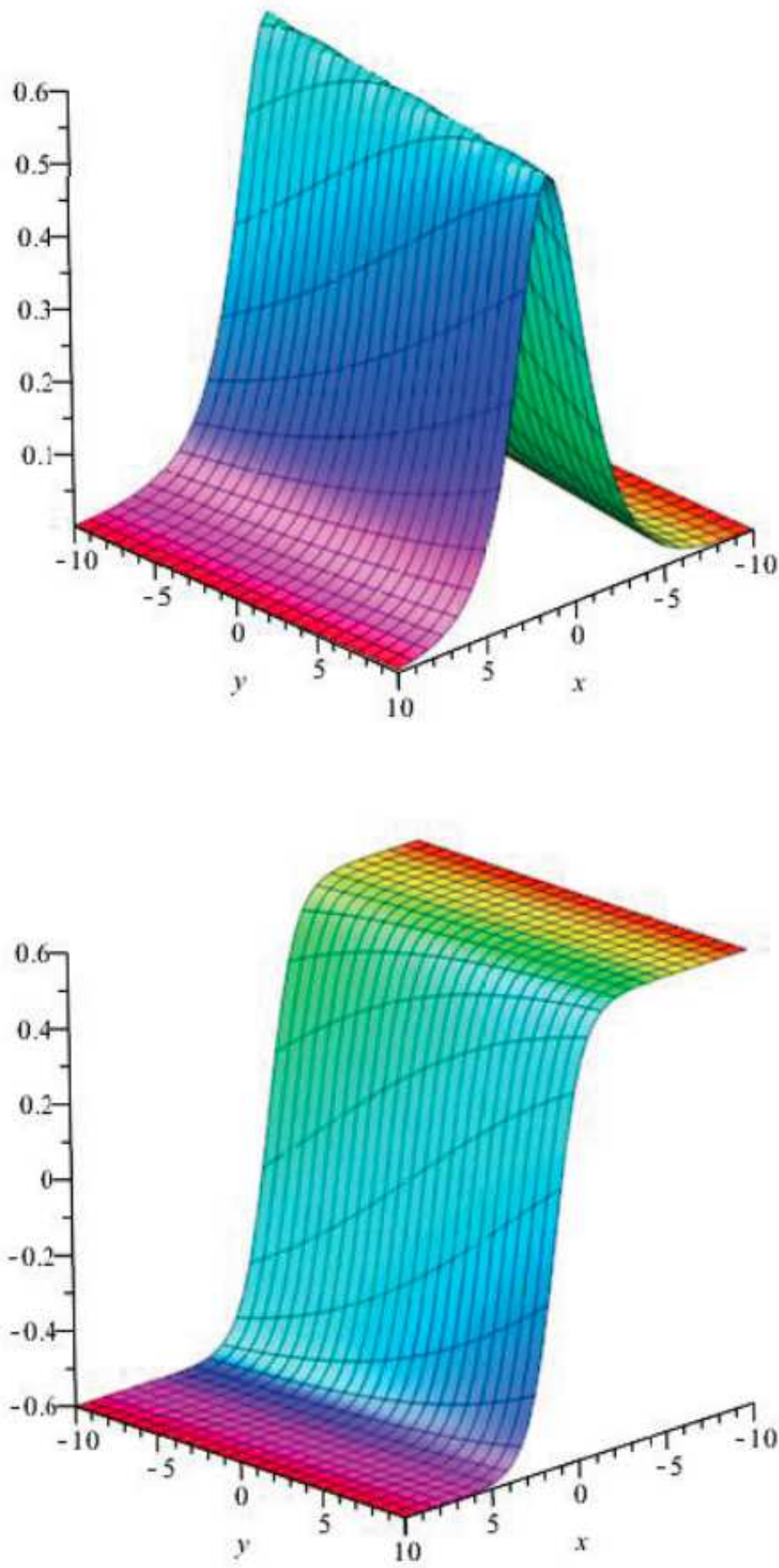

(b)

Figure 5

Please see the Manuscript PDF file for the complete figure caption 